\title{
Investigating lymphocyte populations in patients with Parkinson's disease
}

\author{
Nicolas Dzamko ${ }^{1,2}$ \\ ${ }^{1}$ Central Clinical School, Faculty of Medicine, University of Sydney, Camperdown, NSW, Australia; ${ }^{2}$ School of Medical Sciences, Faculty of \\ Medicine, University of New South Wales, Kensington, NSW, Australia \\ Correspondence to: Nicolas Dzamko. Central Clinical School, Faculty of Medicine, University of Sydney, Camperdown, NSW 2050, Australia. \\ Email: nicolas.dzamko@sydney.edu.au. \\ Provenance and Peer Review: This article was commissioned by the Editorial Office, Annals of Translational Medicine. The article did not undergo \\ external peer review. \\ Comment on: Sun C, Zhao Z, Yu W, et al. Abnormal subpopulations of peripheral blood lymphocytes are involved in Parkinson's disease. Ann Transl \\ Med 2019;7:637.
}

Submitted Jan 23, 2020. Accepted for publication Feb 25, 2020.

doi: 10.21037/atm.2020.02.29

View this article at: http://dx.doi.org/10.21037/atm.2020.02.29

Parkinson's disease (PD) is a globally prevalent age-related neurodegenerative movement disorder. With an average age of onset of 60-70 years, symptoms of PD include muscle rigidity, tremor, bradykinesia, hyposmia, sleep disorders and gastrointestinal dysfunction. PD symptomology is associated with the progressive degeneration of dopamine producing neurons in the midbrain, in conjunction with the spread and pathological accumulation of $\alpha$-synuclein protein in many brain regions (1). PD is progressively disabling and often culminates in dementia and a reduced quality of life in later years. The motor features of PD can be symptomatically treated with dopamine replacement therapy, and for a subset of eligible patients that can tolerate a more invasive approach, deep brain stimulation. However, current therapies do not alleviate non-motor symptoms and they do not slow down or prevent disease progression.

The current lack of disease modifying therapies for PD largely stems from the incomplete understanding of the biology underlying disease pathogenesis. Indeed, by the time PD is clinically diagnosed, approximately $40-60 \%$ of dopamine neurons have already irreversibly degenerated. Estimates based on the extrapolation of brain pathology suggest that PD may commence up to 20 years prior to motor symptoms manifesting and subsequent clinical diagnosis (2). PD symptomology is also heterogenous, confounding both diagnosis and prognosis and suggesting a disease of complex etiology (1). This is supported by genetic studies that collectively suggest a dynamic interplay between genes and the environment may modulate both $\mathrm{PD}$ risk and disease progression [for review see (3)]. As a main interface between the body and the environment, there is increasing interest in the role of the immune system in PD.

Intriguingly, the earliest PD symptoms and earliest signs of $\alpha$-synuclein pathology appear not in the brain, but rather in the periphery (4). Although challenging to measure, pathological $\alpha$-synuclein deposits have been observed in the gastrointestinal tract (5), submandibular gland (6) and skin (7) of prodromal PD patients and/or asymptomatic patients at high risk of developing $\mathrm{PD}$, such as patients with idiopathic REM sleep behaviour disorder (8). Accumulation of $\alpha$-synuclein in the gastrointestinal tract may underlie enteric nervous system dysfunction and subsequent common prodromal PD symptoms such as constipation (9). Moreover, pathological forms of a-synuclein have been shown to activate innate immune inflammatory pathways (10) and modulate $\mathrm{T}$ cell responses (11), ostensibly linking the immune system to PD pathology. Despite this link, determining the extent of immune dysfunction in PD has remained challenging. A number of studies have reported higher levels of inflammatory cytokines in PD patient blood samples, but this is not always observed, and like the clinical symptoms of PD results show considerable heterogeneity [for review see (12)]. A number of studies have also assessed the extent to which circulating immune cell populations in PD patients may underlie inflammatory phenotypes. In general PD is associated with a decrease 
in T lymphocytes, but study outcomes are variable and interpretation complicated by the diversity of lymphocyte subsets and how they are phenotyped [for review see (13)]. Further complicating is that, in line with a slow progressing age related disorder, immune phenotypes in PD patients are subtle, requiring robust sample sizes and careful analysis of the data. Moreover, it is possible that only distinct subsets of patients may display altered lymphocyte populations and this may relate to the heterogenous progression of $\mathrm{PD}$.

To further explore peripheral immune cell populations in the context of PD and its symptomology, Sun and colleagues used flow cytometry to measure the percentage of different lymphocyte populations in blood samples obtained from 127 PD patients and 148 well matched healthy control participants. In comparison to the control cohort, they found a significant increase in natural killer (NK) cells combined with a significant decrease in CD4 $\mathrm{T}$ cells in the PD patients. Both of these findings are in agreement with a meta-analysis of prior studies investigating lymphocyte populations in PD (14). However, an intriguing observation was that the percentages of measured lymphocyte populations had a markedly increased range in the PD patients. The large sample size studied allowed the authors to determine a normal reference range for the measured lymphocyte populations in their specific cohort. Approximately 30\% of PD patients differed from the normal reference range for all lymphocyte populations measured. In all instances, this was significantly higher than the $\sim 10 \%$ of control participants that differed from the normal reference range. Statistical modelling suggested that deviation of NK and CD4 T cells from the reference range was associated with increased PD risk, and patients with a divergent population of CD8 $\mathrm{T}$ cells had worse PD motor symptoms compared to patients in the normal range. These results add to evidence that alterations in the peripheral immune system can influence disease onset and progression, at least in a subset of individuals.

These findings are of interest and certainly raise a number of questions. First is how might altered $\mathrm{T}$ cell populations modulate disease progression? The PD patients that deviate from the normal reference range presumably include members with both abnormally high, and abnormally low percentages of $\mathrm{T}$ cells, thus a direct relationship between lymphocyte numbers and disease progression seems unclear. The authors plausibly suggest, that as has been observed in animal models (15), increased infiltration of $\mathrm{T}$ cells into the brain may promote neuroinflammation and dopamine neuron degeneration. However, this is difficult to prove in a clinical population. It is also possible that factors secreted from immune cells, that more readily cross the blood brain barrier than the immune cells themselves, may influence neuroinflammation and PD progression. At least one example of such a result was recently observed for mice with the PD implicated LRRK2 G2019S mutation (16). Whether there are any phenotypic differences that functionally link the response of the altered immune cell populations to environmental stimuli would be interesting to determine. There is also the question of when do immune cell populations become altered in $\mathrm{PD}$ ? The authors propose that the increased PD risk associated with divergence of certain immune cell populations from the normal range infers potential causality. However, the study of a manifesting clinical PD population always raises questions about cause or consequence. It would certainly be of interest to determine if the same immune cell alterations are observed in de novo PD patients. In particular, it would be of interest to determine if the same results are observed in cohorts at high risk for developing PD in the future, such as carriers of GBA or LRRK2 missense mutations, or patients diagnosed with idiopathic REM sleep behaviour disorder. Complicating such studies though is the relatively lower available sample size, and as the authors explain, the normal reference range for each cohort may be influenced by age, gender, ethnicity, geographical location and experimental methodology. Potentially even more important is longitudinal assessment. Are altered immune cell populations transient, potentially reflecting an acute change to environmental stimuli, or are these stable adaptations with utility as biomarkers and how do the populations change dynamically with time or disease progression? That not all PD patients exhibit the altered immune cell population phenotype also suggests that this is not a singular cause of PD, but rather modulates risk and progression in a distinct subgroup and may contribute to the heterogeneity of PD symptomology. Finally, in agreement with the authors, is that the characterisation of the immune cell populations is potentially only the starting point. For example, within the lymphocyte population is the divergence from the reference range mediated by particular immune cell subsets such as regulatory $\mathrm{T}$ or $\mathrm{B}$ cells? Are the results indicating defects in immune cell differentiation and/or can differences be detected at the level of progenitor cells? Are any defects intrinsic to lymphocytes or potentially mediated by other cells such as myeloid cells? Whether myeloid cells show the same phenotype in PD patients would seem important to assess, given the reports of monocyte dysfunction in PD (17), the role of monocytes in mediating the activation and recruitment of lymphocytes and that 
monocytes are highly enriched in PD implicated genes (18).

In conclusion, the work of Sun and colleagues provides impetus for continued studies into the role of the peripheral immune system in PD, and the potential use of immune readouts for much needed PD biomarkers. As for all clinical studies, the extent to which the results are replicated and how applicable they are across divergent cohorts will be important to determine.

\section{Acknowledgments}

Funding: ND receives funding for Parkinson's disease research from the University of Sydney, Michael J Fox Foundation for Parkinson's disease research, Shake It Up Australia Foundation and the National Health and Medical Research Council.

\section{Footnote}

Conflicts of Interest: The author has no conflicts of interest to declare.

Ethical Statement: The author is accountable for all aspects of the work in ensuring that questions related to the accuracy or integrity of any part of the work are appropriately investigated and resolved.

Open Access Statement: This is an Open Access article distributed in accordance with the Creative Commons Attribution-NonCommercial-NoDerivs 4.0 International License (CC BY-NC-ND 4.0), which permits the noncommercial replication and distribution of the article with the strict proviso that no changes or edits are made and the original work is properly cited (including links to both the formal publication through the relevant DOI and the license). See: https://creativecommons.org/licenses/by-nc-nd/4.0/.

\section{References}

1. Kalia LV, Lang AE. Parkinson's disease. Lancet 2015;386:896-912.

2. Gaig C, Tolosa E. When does Parkinson's disease begin? Mov Disord 2009;24 Suppl 2:S656-64.

3. Cannon JR, Greenamyre JT. Gene-environment interactions in Parkinson's disease: specific evidence in humans and mammalian models. Neurobiol Dis 2013;57:38-46.

4. Braak H, de Vos RA, Bohl J, et al. Gastric alpha-synuclein immunoreactive inclusions in Meissner's and Auerbach's plexuses in cases staged for Parkinson's disease-related brain pathology. Neurosci Lett 2006;396:67-72.

5. Stokholm MG, Danielsen EH, Hamilton-Dutoit SJ, et al. Pathological alpha-synuclein in gastrointestinal tissues from prodromal Parkinson disease patients. Ann Neurol 2016;79:940-9.

6. Vilas D, Iranzo A, Tolosa E, et al. Assessment of alphasynuclein in submandibular glands of patients with idiopathic rapid-eye-movement sleep behaviour disorder: a case-control study. Lancet Neurol 2016;15:708-18.

7. Doppler K, Jentschke HM, Schulmeyer L, et al. Dermal phospho-alpha-synuclein deposits confirm REM sleep behaviour disorder as prodromal Parkinson's disease. Acta Neuropathol 2017;133:535-45.

8. Postuma RB, Gagnon JF, Bertrand JA, et al. Parkinson risk in idiopathic REM sleep behavior disorder: preparing for neuroprotective trials. Neurology 2015;84:1104-13.

9. Stirpe P, Hoffman M, Badiali D, et al. Constipation: an emerging risk factor for Parkinson's disease? Eur J Neurol 2016;23:1606-13.

10. Beraud D, Maguire-Zeiss KA. Misfolded alpha-synuclein and Toll-like receptors: therapeutic targets for Parkinson's disease. Parkinsonism Relat Disord 2012;18 Suppl 1:S17-20.

11. Sulzer D, Alcalay RN, Garretti F, et al. T cells from patients with Parkinson's disease recognize alpha-synuclein peptides. Nature 2017;546:656-61.

12. Dzamko N, Geczy CL, Halliday GM. Inflammation is genetically implicated in Parkinson's disease. Neuroscience 2015;302:89-102.

13. Garretti F, Agalliu D, Lindestam Arlehamn CS, et al. Autoimmunity in Parkinson's Disease: The Role of alphaSynuclein-Specific T Cells. Front Immunol 2019;10:303.

14. Jiang S, Gao H, Luo Q, et al. The correlation of lymphocyte subsets, natural killer cell, and Parkinson's disease: a meta-analysis. Neurol Sci 2017;38:1373-80.

15. Brochard V, Combadiere B, Prigent A, et al. Infiltration of CD4+ lymphocytes into the brain contributes to neurodegeneration in a mouse model of Parkinson disease. J Clin Invest 2009; 119:182-92.

16. Kozina E, Sadasivan S, Jiao Y, et al. Mutant LRRK2 mediates peripheral and central immune responses leading to neurodegeneration in vivo. Brain 2018;141:1753-69.

17. Grozdanov V, Bliederhaeuser C, Ruf WP, et al. Inflammatory dysregulation of blood monocytes in Parkinson's disease patients. Acta Neuropathol 2014;128:651-63.

18. Raj T, Rothamel K, Mostafavi S, et al. Polarization of the effects of autoimmune and neurodegenerative risk alleles in leukocytes. Science 2014;344:519-23.

Cite this article as: Dzamko N. Investigating lymphocyte populations in patients with Parkinson's disease. Ann Transl Med 2020;8(6):276. doi: 10.21037/atm.2020.02.29 